\title{
Influencia del nivel de riesgo perinatal sobre el desarrollo motor grueso de los niños prematuros: Estudio observacional de corte transversal
}

\author{
Francisco Javier Fernández ${ }^{1,2}$, Inmaculada Calvo ${ }^{3,4}$, \\ Sergio Montero ${ }^{3}$, y Galaad Torró ${ }^{1}$ \\ ${ }^{1}$ Centro de Desarrollo Infantil y Atención Temprana (Murcia); ${ }^{2}$ Universidad de Murcia; \\ ${ }^{3}$ Consejería de Educación y Cultura; ${ }^{4}$ Universidad Católica \\ San Antonio de Murcia (España)
}

\begin{abstract}
La prematuridad es uno de los principales problemas de la sanidad perinatal; teniendo en cuenta su elevada incidencia, la morbilidad mostrada a corto y largo plazo, y la escasa evidencia científica que determine la influencia que los factores de riesgo tienen sobre el desarrollo motor en prematuros, nos planteamos realizar un estudio que analizara la influencia que los factores de riesgo perinatales tienen sobre la motricidad gruesa de esta población. El método es un estudio observacional de corte transversal sobre una muestra de 261 prematuros, evaluados desde noviembre de 2014 hasta abril de 2018. Se utilizó la Escala de Desarrollo Motor Infantil de Alberta (AIMS), para comprobar el nivel de desarrollo motor grueso, el percentil en el cual se encuentra el prematuro, y el Inventario de Riesgo Perinatal (PERI) para determinar la puntuación y el nivel de riesgo perinatal. Los resultados encontrados han sido: se muestra una relación significativa e inversa entre el riesgo perinatal y la motricidad gruesa, en ambos sexos $(p=0.000)$. Por tanto, el desarrollo motor grueso de los niños prematuros es menor conforme aumenta el nivel de riesgo perinatal. La hemorragia intracraneal y la edad gestacional son los factores de riesgo perinatales que mayor influencia han mostrado sobre el desarrollo motor grueso de los niños prematuros.
\end{abstract}

Palabras clave: Prematuro, desarrollo motor grueso, factores de riesgo, estudio observacional.

Effect of perinatal risk factors on gross motor development of preterm infants. Cross-sectional observational study. Prematurity is one of the main issue of perinatal health; taking into account its high incidence, the morbidity shown in the short and long term and the scarce scientific evidence that determines the influence that risk factors have on motor development in preterm infants, we propose to conduct a study that will analyse the influence that perinatal factors risk have on the gross motor development of this population. Method is based on: cross-sectional observational study on a sample of 261 preterm infants, evaluated from November 2014 to April 2018. The Alberta Infant Motor Scale (AIMS) was used to assess the level of gross motor development, the percentile in which is the premature one and the Perinatal Risk Inventory (PERI) to determine the score and the level of perinatal risk. Results are a significant and inverse relationship between perinatal risk and gross motor skills is found in both sexes $(p=0.000)$. So, the gross motor development of preterm infants is lower as the level of perinatal risk increases. Intracranial hemorrhage and gestational age are the perinatal risk factors that have shown the greatest influence on the gross motor development of preterm infants.

Keywords: Preterm infants, gross motor development, perinatal risk, observational study.

Correspondencia: Francisco Javier Fernández Rego. Universidad de Murcia, Departamento de Fisioterapia. Avda. Juan Carlos I no 86. C.P.: 30800. Lorca, Murcia (España). E-mail: fjfernan@um.es 
En la actualidad, la prematuridad presenta una elevada incidencia y es uno de los principales problemas de la sanidad perinatal, por la gran morbilidad y mortalidad que conlleva, además de los costes socioeconómicos (Mendoza-Tascón, Claros-Benítez, Mendoza-Tascón, Arias-Guatibonza, y Peñaranda-Ospina, 2016; Piñero, Pérez, Vargas, y Candela, 2014). Esta elevada incidencia de nacimientos pretérmino, ha ocasionado un aumento de la investigación e intervención de los profesionales expertos en el ámbito de la pediatría (Marín-Gabriel et al., 2009).

Según la OMS, el $11,1 \%$ de los recién nacidos, nacen prematuramente cada año. Las tasas de prematuridad más elevadas se concentraron en Asia Meridional (13,3\%), y en África Subsahariana (12,3\%) (Organización Mundial de la Salud, 2012; Blencowe et al., 2012). Por el contrario, las tasas más bajas se localizaron en Europa (6,2\%) (Beck et al., 2010). Aproximadamente el $85 \%$ de los recién nacidos con peso menor de 1500 gramos sobreviven como resultado de los avances tecnológicos y terapéuticos de los últimos años, lo que ha llevado a una mayor preocupación por su desarrollo neurológico (de Kieviet, Zoetebier, van Elburg, Vermeulen, y Oosterlaan, 2012; Ment y Constable, 2007). Entre los supervivientes, hasta el 10\% desarrollan posteriormente deficiencias neurológicas y sensoriales mayores que incluyen alteraciones motoras espásticas, retraso mental, parálisis cerebral (Oskoui, Coutinho, Dykeman, Jette, y Pringsheim, 2013), daño visual o auditivo (Leversen et al., 2011) y de un $50 \%$ a $60 \%$ alteraciones cognitivas, de conducta y problemas de aprendizaje (Kinney et al., 2012).

Además del impacto de nacer antes de tiempo, debemos atender a los diferentes factores de riesgo biológicos y sociales que afectan de forma directa e indirectamente en el desarrollo (Janssen, Nijhuis-van der Sanden, Akkermans, Oostendorp, y Kollée, 2008), produciendo una variación del $4 \%$ al $12 \%$ en los componentes del neurodesarrollo del pretérmino (Heineman et al., 2010). El control de los diferentes factores de riesgo y el adecuado seguimiento de los prematuros con el objetivo de detectar cualquier alteración o retraso, puede ayudar a beneficiarlos de una intervención temprana, cuando el sistema nervioso central tiene una gran neuroplasticidad.

Teniendo en cuenta la elevada incidencia de la prematuridad y la morbilidad mostrada a corto y largo plazo, y la escasa evidencia científica publicada que determine la influencia que los distintos factores de riesgo tienen sobre el desarrollo motor de los niños prematuros, nos planteamos realizar un estudio observacional de corte transversal que analizara la influencia que los factores de riesgo perinatales tienen sobre la motricidad gruesa de esta población. En este sentido, los objetivos de nuestro estudio irán encaminados a determinar el efecto que los factores de riesgo perinatales, medidos con el Inventario de Riesgo Perinatal (PERI), tienen sobre la motricidad gruesa de los niños prematuros, según la puntuación obtenida en la Escala de Desarrollo Motor 
Infantil de Alberta (AIMS); y a conocer los factores de riesgo perinatales que influyen en la motricidad gruesa, medida con la AIMS, en función del trimestre de exploración y del sexo del prematuro.

\section{MÉTODO}

Estudio observacional de corte transversal sobre una muestra de prematuros, evaluados en el Centro de Desarrollo Infantil y Atención Temprana (CDIAT) "Fina Navarro López" del Ayuntamiento de Lorca y en el Hospital Clínico Universitario Virgen de la Arrixaca (HUVA), de la Región de Murcia.

\section{Participantes}

La muestra está formada por 261 neonatos, evaluados en el CDIAT y en el HUVA, desde noviembre de 2014 hasta abril de 2018. Los criterios de inclusión utilizados fueron: a) Recién nacidos con edad gestacional inferior a 37 semanas de gestación; b) Consentimiento informado de los padres o tutores legales para la inclusión de los niños en el estudio; c) Disponibilidad para la evaluación del recién nacido; y los criterios de exclusión: diagnóstico de malformaciones congénitas, enfermedades oncológicas, enfermedad neurológica establecida o encefalopatía hipóxico-isquémica, síndromes y enfermedades metabólicas. La muestra finalmente fue de 138 prematuros (58\% varones).

Respecto a la edad gestacional, el valor mínimo y máximo es de 24,75 y 36,75 semanas de gestación, respectivamente, con una media de 31,61 $\pm 3,25$ semanas. El grupo más prevalente son los recién nacidos muy prematuros, concretamente el 36,2\% (50 prematuros). En relación al peso al nacer, el valor mínimo y máximo es de 600 y 3.300 gramos, respectivamente, con una media de $1.690,83 \pm 653,87$ gramos. En cuanto a la distribución de la muestra en función del peso al nacer atendiendo a la clasificación de edad gestacional, el grupo mayoritario se encuentra compuesto por $50(36,2 \%)$ recién

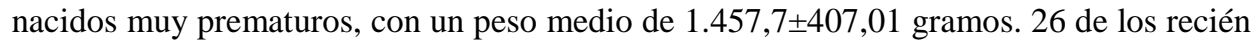
nacidos prematuros extremos (18,8\%) presentan un peso medio de 890,38 $\pm 153,51$ gramos. En relación a la clasificación de la muestra en función del nivel de riesgo, se ha distribuido en tres niveles de riesgo (riesgo bajo, riesgo moderado y riesgo alto) en función de la puntuación total obtenida en el PERI. 78 prematuros $(59,5 \%)$ se encuentran dentro del riesgo considerado como bajo, con una media de 2,57 $\pm 1,99$ en la puntuación obtenida en el PERI. El grupo de prematuros con riesgo alto, compuesto por 32 neonatos $(23,2 \%)$, con una media de $13,68 \pm 3,71$ en la puntuación total del PERI, son los que han sufrido más factores de riesgo a lo largo del periodo perinatal.

Para facilitar el posterior análisis de los resultados, la muestra ha sido estratificada en función del trimestre de vida en el que ha sido evaluado (hasta el sexto 
trimestre). La mayor proporción de prematuros se concentran en el primer, segundo y tercer trimestre de vida, representando el $65,2 \%$, el $12,3 \%$ y el $14,5 \%$ respectivamente. Es importante destacar que, en los nueve primeros meses de vida, es donde ocurren la mayoría de los cambios acaecidos en el desarrollo motor grueso, por este motivo sólo se han tenido en cuenta en el análisis de datos los tres primeros trimestres, aunque es imprescindible conocer qué factores de riesgo pueden tener un mayor impacto en cada uno de los trimestres de vida (Roze, Kerstjens, Maathuis, ter Horst, y Bos, 2008).

\section{Instrumentos}

Para la evaluación se utilizaron dos instrumentos de valoración: la AIMS, para comprobar el nivel de desarrollo motor grueso, y el percentil en el cual se encuentra el prematuro, y el PERI (Piper y Darrah, 1994) para determinar la puntuación y el nivel de riesgo en función del número y gravedad de los factores de riesgo (Scheiner y Sexton, 1991).

\section{Procedimiento}

Una vez evaluado el niño en el CDIAT, se cumplimenta el PERI a partir de los datos contenidos en el informe de alta hospitalaria, obteniendo una puntuación asociada al nivel de riesgo. A continuación, se evalúa el nivel de desarrollo motriz grueso alcanzado por el prematuro, en función de la edad gestacional, mediante la AIMS, obteniendo una puntuación bruta y un percentil de desarrollo. En ocasiones, las valoraciones se realizaron en el HUVA cumplimentando el PERI al nacer o a los pocos días de vida y, posteriormente, determinando el nivel de motricidad gruesa mediante la AIMS.

Las variables utilizadas para el análisis son : a) sexo; b) edad corregida; c) trimestre de evaluación; d) puntuación bruta de la escala AIMS; e) percentil de la escala AIMS; f) edad gestacional; g) clasificación en función de la edad gestacional (cuatro grupos, prematuros extremos: <28 semanas, muy prematuros: 28 a 32 semanas, prematuros moderados: $32+1$ a 34 semanas y prematuros tardíos: $34+1$ a $36+6$ ); h) peso: al nacer (gramos); i) clasificación en función del peso al nacer (cuatro grupos: extremado bajo peso: <1000g, muy bajo peso: $1000 \mathrm{~g}-1499 \mathrm{~g}$, bajo peso: $1500 \mathrm{~g}$ a $2499 \mathrm{~g}$ ); j) variables del PERI (18 variables incluyendo la puntuación total obtenida en el PERI y la puntuación en cada uno de los 18 ítems del propio inventario); k) nivel de riesgo (en función de la puntuación obtenida en el PERI y según las categorías: riesgo bajo:0-6 puntos, moderado: $7-9$ puntos y alto: $>10$ puntos).

Respecto al análisis estadístico, los datos fueron analizados mediante el programa IBM SPSS Statistics for Windows, versión 25.0. (IBM Corp. Released, 2017). Para la clasificación de la muestra, se realizaron análisis descriptivos y análisis de frecuencias. Para el cálculo de los resultados, se utilizaron el ANOVA de un factor, 
correlaciones bivariadas para el cálculo del coeficiente de Pearson y correlaciones bivariadas, para el cálculo del coeficiente de Spearman. Para estandarizar las puntuaciones directas obtenidas con la AIMS, procedimos a transformarlas en puntuaciones $\mathrm{Z}$, basándonos en las medias y desviaciones estándar de la muestra normativa que la propia escala AIMS establece en función de la edad y el sexo (Piper y Darrah, 1994).

En cuanto a los aspectos éticos, durante todo el estudio los investigadores se adhirieron a las Normas de Buena Práctica Clínica y a los principios de la Declaración de Helsinki (World Medical Association, 2013).

\section{RESULTADOS}

Se analizó el efecto del nivel de riesgo perinatal sobre el desarrollo motor grueso de los recién nacidos prematuros en función del sexo y que factores de riesgo muestran mayor efecto en el desarrollo motor grueso en la cohorte a nivel general y en los tres primeros trimestres de evaluación.

Para analizar el efecto del nivel de riesgo sobre la motricidad gruesa, utilizamos el coeficiente de correlación de Spearman, ya que permite determinar si existe asociación significativa entre los tres niveles de riesgo del PERI (riesgo bajo, riesgo moderado y riesgo alto) y la puntuación motora gruesa obtenida en la escala Alberta.

En el caso de los prematuros sin distinción en sexo, la prueba de Spearman ha mostrado resultados estadísticamente significativos $(p=0.000) \mathrm{y}$, por lo tanto, conforme el nivel de riesgo perinatal es mayor en el prematuro, menor es el desarrollo motor grueso alcanzado en la escala AIMS.

Para comprobar cómo afecta el riesgo perinatal a la motricidad gruesa de los prematuros en función del sexo, se utilizó el coeficiente de correlación de Spearman. La tabla 1 recoge los estadísticos descriptivos de la muestra divididos en varones y mujeres y la tabla 2, los resultados de la prueba de Spearman.

Tabla 1. Estadísticos descriptivos de las variables nivel de riesgo y puntuación bruta del AIMS en función del sexo

\begin{tabular}{lrrrrrr}
\hline & $N$ & Media & $\begin{array}{c}\text { Desviación } \\
\text { estándar }\end{array}$ & $\begin{array}{c}\text { Error } \\
\text { estándar }\end{array}$ & Mínimo & Máximo \\
\hline VARONES & & \multicolumn{7}{c}{} & & & \\
\hline Riesgo bajo & 43 & 16.9302 & 16.36401 & 2.49549 & 1.00 & 57.00 \\
\hline Riesgo moderado & 18 & 5.8333 & 3.97418 & .93672 & 3.00 & 21.00 \\
\hline Riesgo alto & 19 & 5.0526 & 2.43752 & .55921 & 2.00 & 12.00 \\
\hline Total & 80 & 11.6125 & 13.43385 & 1.50195 & 1.00 & 57.00 \\
\hline MUJERES & \multicolumn{7}{c}{} & & & & \\
\hline Riesgo bajo & 35 & 15.1143 & 12.36877 & 2.09070 & 3.00 & 49.00 \\
\hline Riesgo moderado & 10 & 4.8000 & 1.68655 & .53333 & 3.00 & 9.00 \\
\hline Riesgo alto & 13 & 6.5385 & 4.92638 & 1.36633 & 2.00 & 18.00 \\
\hline Total & 58 & 11.4138 & 10.87736 & 1.42827 & 2.00 & 49.00 \\
\hline
\end{tabular}


Tabla 2. Prueba de Spearman entre el nivel de riesgo y la puntuación bruta del AIMS en función del sexo

\begin{tabular}{|c|c|c|c|c|}
\hline & & & $\begin{array}{c}\text { Nivel de riesgo en } \\
\text { PERI }\end{array}$ & $\begin{array}{c}\text { Puntuación } \\
\text { bruta }\end{array}$ \\
\hline \multicolumn{5}{|l|}{ VARONES } \\
\hline \multirow{6}{*}{$\begin{array}{l}\text { Rho de } \\
\text { Spearman }\end{array}$} & \multirow{3}{*}{$\begin{array}{c}\text { Nivel de riesgo en } \\
\text { PERI }\end{array}$} & Coeficiente de correlación & 1.000 & -.464 \\
\hline & & Sig. (bilateral) & & .000 \\
\hline & & $N$ & 80 & 80 \\
\hline & \multirow{3}{*}{ Puntuación bruta } & Coeficiente de correlación & -.464 & 1.000 \\
\hline & & Sig. (bilateral) & .000 & . \\
\hline & & $N$ & 80 & 80 \\
\hline \multicolumn{5}{|l|}{ MUJERES } \\
\hline \multirow{6}{*}{$\begin{array}{l}\text { Rho de } \\
\text { Spearman }\end{array}$} & \multirow{3}{*}{ Nivel de riesgo } & Coeficiente de correlación & 1.000 & -.404 \\
\hline & & Sig. (bilateral) & . & .002 \\
\hline & & $\mathrm{N}$ & 58 & 58 \\
\hline & \multirow{3}{*}{ Puntuación bruta } & Coeficiente de correlación & -.404 & 1.000 \\
\hline & & Sig. (bilateral) & .002 & \\
\hline & & $N$ & 58 & 58 \\
\hline
\end{tabular}

A partir de los resultados obtenidos, interpretamos que los niveles de riesgo perinatal se encuentran asociados a la puntuación bruta obtenida en la escala AIMS tanto en varones $(p=0.000)$ como en mujeres $(p=0.002)$. La gráfica 1 describe la distribución observada: conforme el nivel de riesgo perinatal es mayor, menor es el desarrollo motor alcanzado por la población de prematuros, con efecto especialmente importante en varones.

Gráfica 1. Nivel de riesgo y la puntuación bruta del AIMS en función del sexo

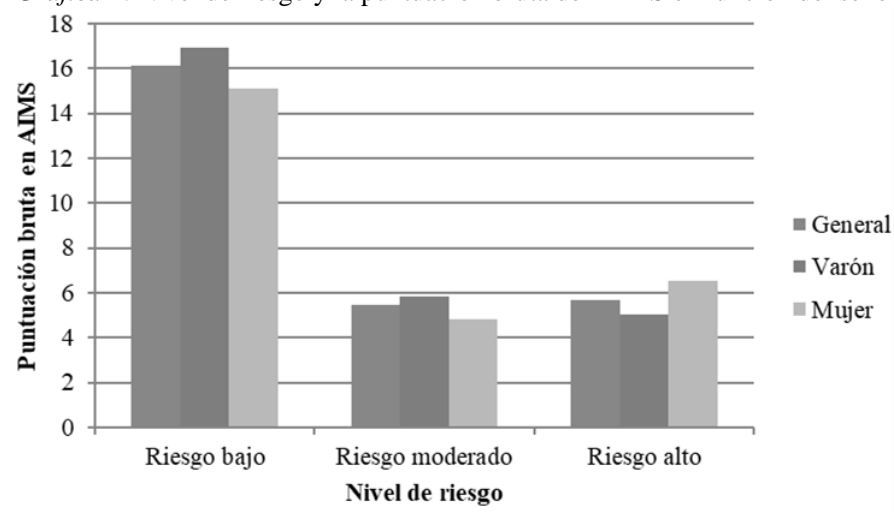

Para analizar los factores de riesgo más influyentes en la motricidad gruesa, se realizó un análisis de regresión lineal por pasos "hacia atrás" utilizando, para ello, la puntuación obtenida en cada uno de los ítems del PERI y, como variable dependiente, el percentil de desarrollo motor grueso, ya que es un valor que nos permite demostrar si el niño prematuro se encuentra fuera o dentro del rango de desarrollo normativo establecido por la propia escala. De la misma forma, los factores de riesgo han sido clasificados en función de los tres primeros trimestres de vida, puesto que son los más 
representativos de la población prematura, y una clasificación general, teniendo en cuenta hasta el sexto trimestre.

En la Tabla 3 se exponen cada uno de los factores de riesgo más influyentes en función del sexo y en función del trimestre de evaluación añadiendo, además, el porcentaje de proporción de varianza para cada caso. Por lo tanto, los factores de riesgo perinatales que mayor efecto van a presentar en el desarrollo motor grueso son la hemorragia intracraneal y las semanas de gestación, justificando el $9.3 \%$ de la varianza encontrada en la puntuación percentil de la escala AIMS.

Los factores de riesgo que se observan con mayor frecuencia en los tres primeros trimestres de evaluación son, principalmente, la hiperbilirrubinemia, el peso al nacer y la necesidad de ventilación mecánica.

Tabla 3. Factores de riesgo más influyentes en función del trimestre de evaluación y el sexo

\begin{tabular}{cccc}
\hline General & $\begin{array}{c}\text { Hemorragia intracraneal } \\
\text { y semanas de gestación } \\
(9.3 \%)\end{array}$ & $\begin{array}{c}\text { Hemorragia intracraneal. } \\
\text { semanas de gestación y } \\
\text { ventilación mecánica } \\
(21.5 \%)\end{array}$ & $\begin{array}{c}\text { Problemas médicos asociados } \\
\text { e infección congénita }(2 \%)\end{array}$ \\
\hline $\begin{array}{c}1^{\circ} \text { trimestre } \\
\text { Hemorragia intracraneal } \\
\text { y semanas de gestación } \\
(12.5 \%)\end{array}$ & $\begin{array}{c}\text { Peso al nacer. hemorragia } \\
\text { intracraneal. semanas de } \\
\text { gestación y ventilación } \\
\text { mecánica }(31.1 \%)\end{array}$ & $\begin{array}{c}\text { Hiperbilirrubinemia y } \\
\text { ventilación mecánica }(6.3 \%)\end{array}$ \\
\hline trimestre & $\begin{array}{c}\text { Problemas médicos } \\
\text { asociados y ventilación } \\
\text { mecánica }(23.1 \%)\end{array}$ & $\begin{array}{c}\text { Peso al nacer e } \\
\text { hiperbilirrubinemia }(28.7 \%)\end{array}$ & $\begin{array}{c}\text { Problemas médicos asociados. } \\
\text { hiperbilirrubinemia. peso al } \\
\text { nacer. infección congénita y } \\
\text { semanas de gestación }(25.4 \%)\end{array}$ \\
\hline $3^{\circ}$ trimestre & $\begin{array}{c}\text { Peso al nacer y } \\
\text { ventilación mecánica } \\
(2.6 \%)\end{array}$ & $\begin{array}{c}\text { Hipoglucemia y peso al } \\
\text { nacer }(0.5 \%)\end{array}$ & $\begin{array}{c}\text { Hiperbilirrubinemia e } \\
\text { infección congénita }(67.4 \%)\end{array}$ \\
\hline
\end{tabular}

Durante el primer trimestre de evaluación, los factores de riesgo perinatales que han mostrado una mayor capacidad predictiva sobre el desarrollo motor son: la hemorragia intracraneal, la ventilación mecánica y las semanas de gestación. Sin embargo, en el tercer trimestre, los factores más destacados por su capacidad predictiva son: la hiperbilirrubinemia, el peso al nacer y la ventilación mecánica.

Conforme pasan los meses y el niño es evaluado por primera vez, a mayor edad, los problemas médicos asociados, la hiperbilirrubinemia y el peso al nacer son los factores de riesgo que mejor predicen los resultados del desarrollo motor grueso de los prematuros.

\section{DISCUSIÓN}

Los objetivos de esta investigación fueron comprobar el efecto del nivel de riesgo perinatal del PERI sobre la motricidad gruesa, según la puntuación obtenida en la AIMS, y conocer cuáles de los factores de riesgo perinatales influyen en la motricidad gruesa, en función del trimestre de evaluación y del sexo del pretérmino. 
En relación a cómo afecta el nivel de riesgo perinatal sobre la motricidad gruesa de los niños prematuros, los resultados muestran una relación significativa e inversa entre ambas variables, tanto para el grupo de varones como para el de mujeres. En relación a la determinación de cuáles son los factores de riesgo perinatales que presentan un mayor efecto sobre la motricidad gruesa de los prematuros en los diferentes trimestres, los resultados indican que la hemorragia intracraneal y las semanas de gestación son los factores que mejor pronostican el desarrollo motor grueso medidos con la escala AIMS.

Para analizar los datos obtenidos con otras investigaciones previas, dividiremos, por un lado, el efecto del riesgo perinatal sobre la motricidad gruesa y, por otro lado, cuáles son los factores de riesgo que influyen en el desarrollo motor según la evidencia recogida para este objetivo.

Son pocos los estudios que evalúan el efecto conjunto de los factores de riesgo sobre el desarrollo motor. Algunos investigadores afirman que el patrón de anormalidad en el desarrollo difiere en función de la exposición a factores perinatales (Brown, Doyle, Bear, y Inder, 2006) y, por tanto, conforme se van acumulando mayor número de factores de riesgo, más peligro existe de mostrar un retraso en las adquisiciones motoras, especialmente si estos factores son graves (Heineman et al., 2010).

El estudio de Pritchard et al. (2013), muestra que el efecto sobre el desarrollo motor se encuentra dividido en función de los siguientes porcentajes: $50 \%$ factores perinatales, $34 \%$ factores psicosociales y $19 \%$ factores sociales. Además, indican que la sensibilidad de los factores de riesgo perinatales es del $70 \%$ para los prematuros con alteraciones en el desarrollo a los 12 meses, aunque la especificidad es del 52\%. Esta es una de las grandes ventajas del PERI, ya que permite obtener una puntuación total y aproximada del nivel de riesgo perinatal para poder compararla con otras puntuaciones.

A continuación, se describen cada uno de los factores de riesgo perinatales predominantes en el desarrollo motor grueso de los prematuros, teniendo en cuenta los resultados obtenidos y los datos señalados en estudios previos.

La hemorragia intracraneal ha sido considerada uno de los factores perinatales más influyentes en el desarrollo del niño prematuro. Las lesiones atípicas del cerebro del niño pretérmino se expresan con un desarrollo motor diferente a la de los niños prematuros sin patología, especialmente si la gravedad de las mismas aumenta (Lee et al., 2010). Spittle, Boyd, Inder, y Doyle (2009), han observado que las anormalidades en la sustancia blanca y el movimiento general, se encuentran significativamente relacionados con los resultados del desarrollo motor al mes y a los tres meses medidos con la escala AIMS, representando las dos únicas evaluaciones eficaces para detectar retrasos en el desarrollo motor y parálisis cerebral con un $100 \%$ de sensibilidad, y, por tanto, evidencian, una vez más, el valor predictivo que tiene la presencia de lesiones parenquimatosas sobre el desarrollo motor (García et al., 2013). 
Por otro lado, la edad gestacional ha sido considerada como un factor de riesgo perinatal a tener en cuenta durante el desarrollo motor del prematuro (Thomaidis et al., 2014), destacando especialmente a Roze et al. (2008), que indicaron que la edad gestacional es el factor de riesgo más influyente, capaz de mostrar un efecto directo sobre el desarrollo motor de los niños prematuros. Volpe (2009), realizó una aproximación causal al motivo de por qué la edad gestacional afecta al desarrollo motor, señalando que, el 50\% de los prematuros nacidos antes de las 29 semanas de gestación desarrollan problemas neurológicos conocidos como "encefalopatía del prematuro", que consta principalmente de dos fases: inicialmente, se produce un daño hipóxicoisquémico donde mueren una gran cantidad de oligodendrocitos y neuronas $\mathrm{y}$, seguidamente, se produce un nuevo desarrollo y migración de neuronas. El resto de factores perinatales, van a provocar un efecto relativo de forma alternativa en cada uno de los meses de evolución del niño prematuro.

Así mismo, el ductus arterioso persistente, las infecciones nosocomiales o no congénitas, la retinopatía del prematuro y la enterocolitis necrotizante son patologías muy significativas en el desarrollo del niño prematuro que, en contraposición, se encuentran reunidas exclusivamente en el ítem "problemas médicos asociados" del Inventario de Riesgo Perinatal, alcanzando, como máximo, una puntuación de 3 en dicho ítem, independientemente del total de patologías que reúna y de la gravedad de las mismas.

Existe una gran variedad de artículos que muestran el efecto de estas cuatro patologías sobre el desarrollo motor de los neonatos prematuros, (Brown et al., 2006; Ferreira, Mello, y Silva, 2014; Roze et al., 2008) destacando, por ejemplo, el estudio de Christian et al. (2014), donde se indica que la sepsis fue un factor con un alto grado de asociación a bajas puntuaciones en los test cognitivos y motores e, igualmente, el estudio de Zanudin et al. (2013) donde la enterocolitis necrotizante fue uno de los factores asociados a un bajo desarrollo motor a los 18 meses, junto con la hemorragia intracraneal y la displasia broncopulmonar.

Finalmente, algunos estudios han incluido la ventilación mecánica como un factor de riesgo perinatal a tener en cuenta para la evaluación motora de los niños prematuros (Brown et al., 2006; Janssen et al., 2008; Potharts et al., 2013).

Una limitación, a tener en cuenta, en relación al PERI es la ausencia de factores relacionados con el ambiente psicosocial del prematuro, especialmente factores asociados a los padres, donde una gran multitud de estudios han corroborado el efecto de estos factores en el desarrollo motor, cognitivo y conductual de los neonatos prematuros (Bos, Einspieler, y Prechtl, 2001; Janssen et al., 2008; Potharts et al., 2013) con un efecto aproximado del 34\% según el estudio de Pritchard et al. (2013).

Saber cuáles de los factores de riesgo perinatales presentan un efecto sobre el desarrollo motor grueso de los niños prematuros nos permite conocer qué niños son 
susceptibles de ser seguidos por riesgo de presentar alteraciones motoras futuras. En este sentido, el PERI nos indica un nivel asociado de riesgo que se relaciona de forma directa con el desarrollo motor grueso, pudiendo prever cuáles son las limitaciones del prematuro en relación a este desarrollo.

Las principales limitaciones de este estudio han sido la falta de homogeneidad de la muestra de estudio en función del sexo, los condicionantes del nacimiento prematuro y la morbilidad asociada, lo que puede dificultar la generalización de los resultados.

Futuras investigaciones deberían tener en cuenta los factores psicosociales y su efecto sobre el desarrollo motor grueso. Además, el PERI, aunque es un instrumento adecuado para el análisis de los factores biológicos de riesgo, es poco discriminativo en relación a determinados factores, así mismo, el ítem "problemas médicos asociados" reúne varias patologías asociadas al retraso en el desarrollo motor, limitando su posterior análisis como factores de riesgo aislados. Por tanto, la mejora del PERI constituye, en sí misma, una futura línea de investigación.

\section{CONCLUSIONES}

El desarrollo motor grueso de los niños prematuros es menor conforme aumenta el nivel de riesgo perinatal, en ambos sexos.

La hemorragia intracraneal y la edad gestacional son los factores de riesgo perinatales que mayor influencia han mostrado sobre el desarrollo motor grueso de los niños prematuros en los tres primeros meses de edad corregida. El peso al nacer, la hemorragia intracraneal, las semanas de gestación y la ventilación mecánica afectan principalmente al sexo masculino en el primer trimestre, mientras que la hiperbilirrubinemia y la ventilación mecánica afectan más al desarrollo motor grueso de las niñas en este trimestre.

En el segundo trimestre el desarrollo motor grueso de los varones se ve más afectado por el peso al nacer y la hiperbilirrubinemia mientras que las niñas prematuras son más sensibles a los problemas médicos asociados, la hiperbilirrubinemia, el peso al nacer, la infección congénita y las semanas de gestación. En el tercer trimestre los factores más influyentes en el desarrollo motor grueso de los niños prematuros son la hipoglucemia y el peso al nacer y en el caso de las niñas la hiperbilirrubinemia y la infección congénita.

\section{REFERENCIAS}

Beck, S., Wojdyla, D., Say, L., Betran, A.P., Merialdi, M., Requejo, J.H., ... Van Look, P.F.A. (2010). The worldwide incidence of preterm birth: a systematic review of maternal mortality and morbidity. Bulletin of the World Health Organization, 88(1), 31-38. 
Blencowe, H., Cousens, S., Oestergaard, M.Z., Chou, D., Moller, A.B., Narwal, R., ... Lawn, J.E. (2012). National, regional, and worldwide estimates of preterm birth rates in the year 2010 with time trends since 1990 for selected countries: a systematic analysis and implications. Lancet, 379(9832), 2162-2172.

Bos, A.F., Einspieler, C., y Prechtl, H.F. (2001). Intrauterine growth retardation, general movements, and neurodevelopmental outcome: a review. Developmental Medicine and Child Neurology, 43(1), 61-68.

Brown, N.C., Doyle, L.W., Bear, M.J., y Inder, T.E. (2006). Alterations in neurobehavior at term reflect differing perinatal exposures in very preterm infants. Pediatrics, 118(6), 2461-2471.

Christian, P., Murray-Kolb, L.E., Tielsch, J.M., Katz, J., LeClerq, S.C., y Khatry, S.K. (2014). Associations between preterm birth, small-for-gestational age, and neonatal morbidity and cognitive function among school-age children in Nepal. BMC Pediatrics, 14, 58-66.

de Kieviet, J.F., Zoetebier, L., van Elburg, R.M., Vermeulen, R.J., y Oosterlaan, J. (2012). Brain development of very preterm and very low-birthweight children in childhood and adolescence: a meta-analysis. Developmental Medicine and Child Neurology, 54(4), 313-323.

Fernández-Rego, FJ. (2014). Fisioterapia en neonatología. En Piñero, J., Pérez, J., Vargas, F., y Candela, A. (Eds). Atención Temprana en el Ámbito Hospitalario (pp. 189-236). Madrid, España: Ediciones Pirámide.

Ferreira, R.C., Mello, R.R., y Silva, K.S. (2014). Neonatal sepsis as a risk factor for neurodevelopmental changes in preterm infants with very low birth weight. Jornal de Pediatría, 90(3), 293-299.

García, P., San Feliciano, L., Benito, F., García, R., Guzmán, J., Salas, S., ... Figueras, J. (2013). Outcome at two years corrected age of a cohort of very low birth weight infants from hospitals within the neonatal SEN1500 network. Anales de Pediatría, 79(5), 279-287.

Heineman, K.R., La Bastide-Van, S., Fidler, V., Middelburg, K.J., Bos, A.F., y Hadders-Algra, M. (2010). Construct validity of the Infant Motor Profile: relation with prenatal, perinatal, and neonatal risk factors. Developmental Medicine and Child Neurology, 52(9), e209e215.

IBM Corp. Released (2017). IBM SPSS Statistics for Windows, Version 25.0. Armonk, New York: IBM Corp.

Janssen, A.J.W.M., Nijhuis-van der Sanden, M.W.G., Akkermans, R.P., Oostendorp, R.B., y Kollée, L.A.A. (2008). Influence of behaviour and risk factors on motor performance in preterm infants at age 2 to 3 years. Developmental Medicine and Child Neurology, 50(12), 926-931.

Kinney, H.C., Haynes, R.L., Xu, G., Andiman, S.E., Folkerth, R.D., Sleeper, L.A., y Volpe, J.J. (2012). Neuron deficit in the white matter and subplate in periventricular leukomalacia. Annals of Neurology, 71(3), 397-406.

Lee, J.Y., Kim, H.S., Jung, E., Kim, E.S., Shim, G.H., Lee, H.J., ... Choi, J.H. (2010). Risk factors for periventricular-intraventricular hemorrhage in premature infants. Journal of Korean Medical Science, 25(3), 418-424.

Leversen, K.T., Sommerfelt, K., Rønnestad, A., Kaaresen, P.I., Farstad, T., Skranes, J., ... Markestad, T. (2011). Prediction of neurodevelopmental and sensory outcome at 5 years in Norwegian children born extremely preterm. Pediatrics, 127(3), e630-e638.

Marín, M.A., Pallás, C.R., De La Cruz, J., Caserío, S., López, M., Moral, M., ... Lora, D. (2009). Age of sitting unsupported and independent walking in very low birth weight preterm infants with normal motor development at 2 years. Acta Pediátrica, 98(11), 1815-1821. 
Mendoza, L.A., Claros, D.I., Mendoza, L.I., Arias, M.D., y Peñaranda, C.B. (2016). Epidemiología de la prematuridad, sus determinantes y prevención del parto prematuro. Revista Chilena de Obstetricia y Ginecología, 8(4), 330-342.

Ment, L.R., y Constable, R.T. (2007). Injury and recovery in the developing brain: evidence from functional MRI studies of prematurely born children. Nature Clinical Practice Neurology, 3(10), 558-571.

Organización Mundial de la Salud (2012). Nacidos demasiado pronto: informe de acción global sobre nacimientos prematuros. Ginebra: OMS.

Oskoui, M., Coutinho, F., Dykeman, J., Jetté, N., y Pringsheim, T. (2013). An update on the prevalence of cerebral palsy: a systematic review and meta-analysis. Developmental Medicine and Child Neurology, 55(6), 509-519.

Piper, M.C., y Darrah, J. (1994). Motor Assessment of the Developing Infant. Philadelphia, United States: WB Saunders Company.

Potharst, E.S., van Wassenaer-Leemhuis, A.G., Houtzager, B.A., Livesey, D., Kok, J.H., Last, B.F., y Oosterlaan, J. (2013). Perinatal risk factors for neurocognitive impairments in preschool children born very preterm. Developmental Medicine and Child Neurology, 55(2), 178-184.

Pritchard, M.A., Colditz, P.B., Cartwright, D., Gray, P.H., Tudehope, D., y Beller, E. (2013). Risk determinants in early intervention use during the first postnatal year in children born very preterm. BMC Pediatrics, 13, 201-205.

Roze, E., Kerstjens, J.M., Maathuis, C.G.B., ter Horst, H.J., y Bos, A.F. (2008). Risk factors for adverse outcome in preterm infants with periventricular hemorrhagic infarction. Pediatrics, 122(1), e46-e52.

Scheiner, A.P., y Sexton, M.E. (1991). Prediction of developmental outcome using a perinatal risk inventory. Pediatrics, 88(6), 1135-1143.

Spittle, A.J., Boyd, R.N., Inder, T.E., y Doyle, L.W. (2009). Predicting motor development in very preterm infants at 12 months' corrected age: the role of qualitative magnetic resonance imaging and general movements assessments. Pediatrics, 123(2), 512-517.

Thomaidis, L., Zantopoulos, G.Z., Fouzas, S., Mantagou, L., Bakoula, C., y Konstantopoulos, A. (2014). Predictors of severity and outcome of global developmental delay without definitive etiologic yield: a prospective observational study. BMC Pediatrics, 14, 40-47.

Volpe, J.J. (2009). The encephalopathy of prematurity--brain injury and impaired brain development inextricably intertwined. Seminars in Pediatric Neurology, 16(4), 167-178.

World Medical Association (2013). World Medical Association Declaration of Helsinki: ethical principles for medical research involving human subjects. Journal of the American Medical Association, 310(20), 2191-2194.

Zanudin, A., Burns, Y., Gray, P.H., Danks, M., Poulsen, L., y Watter, P. (2013). Perinatal events and motor performance of children born with ELBW and nondisabled. Pediatric Physical Therapy: The Official Publication of the Section on Pediatrics of the American Physical Therapy Association, 25(1), 30-35.

Recibido: 19 de septiembre de 2019 Recepción Modificaciones: 30 de octubre de 2019 Aceptado: 2 de noviembre de 2019 